\title{
Universiteit
}

Leiden

The Netherlands

\section{A compact multipurpose nanomanipulator for use inside a scanning electron microscope}

Heeres, E.C.; Katan, A.J.; Es, M.H. van; Beker, A.F.; Hesselberth, M.B.S.; Zalm, D.J. van der; Oosterkamp, T.H.

\section{Citation}

Heeres, E. C., Katan, A. J., Es, M. H. van, Beker, A. F., Hesselberth, M. B. S., Zalm, D. J. van der, \& Oosterkamp, T. H. (2010). A compact multipurpose nanomanipulator for use inside a scanning electron microscope. Review Of Scientific Instruments, 81, 023704.

doi:10.1063/1.3271033

Version: $\quad$ Not Applicable (or Unknown)

License: $\quad$ Leiden University Non-exclusive license

Downloaded from: https://hdl.handle.net/1887/61346

Note: To cite this publication please use the final published version (if applicable). 


\section{A compact multipurpose nanomanipulator for use inside a scanning electron microscope}

E. C. Heeres, A. J. Katan, M. H. van Es, A. F. Beker, M. Hesselberth, D. J. van der Zalm, and T. H. Oosterkamp

Citation: Review of Scientific Instruments 81, 023704 (2010); doi: 10.1063/1.3271033

View online: http://dx.doi.org/10.1063/1.3271033

View Table of Contents: http://aip.scitation.org/toc/rsi/81/2

Published by the American Institute of Physics

\section{Articles you may be interested in}

A superconducting quantum interference device based read-out of a subattonewton force sensor operating at millikelvin temperatures

Applied Physics Letters 98, 133105 (2011); 10.1063/1.3570628

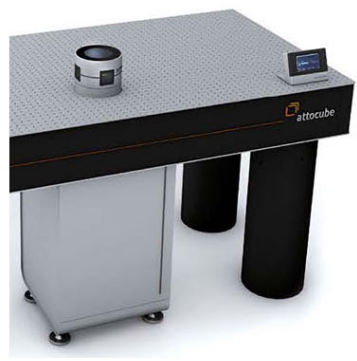

Obstruction free access 


\title{
A compact multipurpose nanomanipulator for use inside a scanning electron microscope
}

\author{
E. C. Heeres, ${ }^{1}$ A. J. Katan, ${ }^{1}$ M. H. van Es, ${ }^{1}$ A. F. Beker, ${ }^{1}$ M. Hesselberth, ${ }^{1}$ \\ D. J. van der Zalm, ${ }^{1}$ and T. H. Oosterkamp ${ }^{1,2}$ \\ ${ }^{1}$ Leiden Institute of Physics, Leiden University, Niels Bohrweg 2, 2333 CA Leiden, The Netherlands \\ ${ }^{2}$ Leiden Probe Microscopy, Niels Bohrweg 2, 2333 CA Leiden, The Netherlands
}

(Received 13 July 2009; accepted 9 November 2009; published online 5 February 2010)

\begin{abstract}
A compact, two-stage nanomanipulator was designed and built for use inside a scanning electron microscope. It consists of a fine stage employing piezostacks that provide a $15 \mu \mathrm{m}$ range in three dimensions and a coarse stage based on commercially available stick-slip motors. Besides the fabrication of enhanced probes for scanning probe microscopy and the enhancement of electron field emitters, other novel manipulation processes were developed, such as locating, picking up, and positioning small nanostructures with an accuracy of $\sim 10 \mathrm{~nm}$. In combination with in situ I-V experiments, welding, and etching, this results in a multipurpose nanofactory, enabling a new range of experiments. () 2010 American Institute of Physics. [doi:10.1063/1.3271033]
\end{abstract}

\section{INTRODUCTION}

Nanomanipulation inside an electron microscope can give control on a very fine scale while providing real time feedback on the object being manipulated. ${ }^{1-3}$ Just as other available scanning electron microscope (SEM) add-ons, such as a gas injection system (GIS), energy dispersive x-ray analysis (EDX) or a variable temperature stage, a nanomanipulator extends the applicability of the electron microscope far beyond an imaging tool. Sample fabrication processes often include characterization and localization of features of interest using optical microscopy, atomic force microscopy (AFM) or SEM and a subsequent design of actuation, measurement or control structures, often by lithographic processes. ${ }^{4,5}$ A drawback of AFM and STM manipulation is that the process cannot be imaged, only the result, as the object to manipulate with is also used to obtain the image. With a manipulator inside a SEM however, the feature of interest can be accurately positioned in situ, immediately after localization onto another predefined structure.

In this paper we first discuss the constraints to our design set by our electron microscope, then we discuss the design considerations that improve the user friendliness of the manipulator, the properties of the manipulator, and finally we give some examples of fabricated structures.

\section{DESIGN CONSIDERATIONS}

To image and manipulate even the smallest nano-objects, such as as-grown single-walled carbon nanotubes lying on a Si substrate, or protruding from the edge of such substrates, we employ a $30 \mathrm{kV}$ SEM (FEI, Nova NanoSEM), which is equipped with a field emission source and a magnetic immersion lens system and has a measured resolution of $1 \mathrm{~nm}$. To reduce the deposition of amorphous carbon during SEM imaging, a plasma cleaner is installed and used to regularly clean the SEM chamber. Because of this it is also necessary to use exclusively ultrahigh vacuum (UHV) compatible ma- terials inside. Such a high-resolution microscope also imposes a number of restrictions on the design of a nanomanipulator to be used inside. Because of its magnetic immersion lens, all materials used in the manipulator have to be nonmagnetic. Due to the size of the chamber, a compact design with a height of less than $57 \mathrm{~mm}$ is needed, such that the manipulator fits in the limited space underneath the final lens and experiments can be performed at the SEM eucentric working distance of $5 \mathrm{~mm}$ (see also Fig. 1). With a total height of $52 \mathrm{~mm}$ for the entire manipulator we can thus work at a maximum working distance of $10 \mathrm{~mm}$ down to the smallest allowable working distance. The entire manipulator can be positioned within the chamber by moving the SEM stage.

To allow a wide variety of experiments, sliders were made that allow manipulation of different types of objects: sharp tips, e.g., etched metallic wires, AFM chips or field emission sources but also flat samples. By using such sliders, the time needed to create a functionalized probe is reduced because it allows the rapid exchange of the tip and/or the sample that contains the objects that are to be mounted. For this too, the SEM is more convenient than a transmission electron microscope (TEM), where sample sizes are restricted to a few millimeters and waiting times are often longer. The sliding system has been designed in such a way that different holders-each designed for a specific tipslide onto the manipulator base. A guiding rail and spring clamping assembly enable a stable but movable connection. Furthermore, the detached slider enables simple installation and positioning of a tip or substrate outside the confined environment of the SEM chamber.

The range of motion of the manipulator should be large enough to be able to preposition the samples manually without the need of an optical microscope. As nanomaterials are often grown onto substrates of several $\mathrm{cm}^{2}$, a range of several millimeters is desirable such that cleaving of the sample is not necessarily needed and a large area can be searched to 

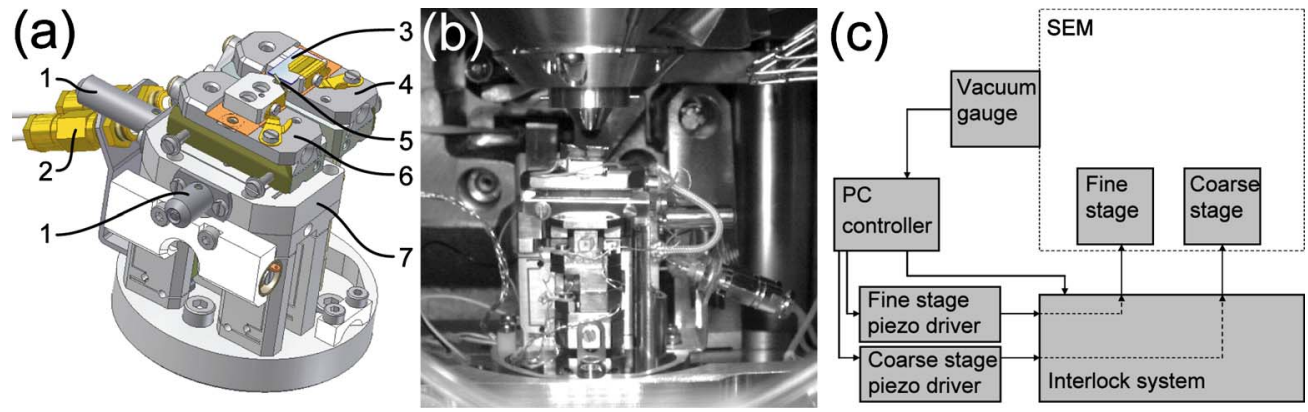

FIG. 1. (Color online) (a) 3D image of the nanomanipulator with the following numbered parts: (1) fine stage piezoactuator, (2) IV connector, (3) flat substrate holding objects to be manipulated (e.g., nanotubes, nanowires, and diamond nanocrystals), (4) flat substrate slider on coarse stage, (5) AFM chip onto or by which objects are manipulated, (6) AFM chip slider on fine stage, (7) fine stage. (b) CCD image of the nanomanipulator installed in the SEM. The total available height underneath the polepiece is $62 \mathrm{~mm}$. To be able to work at eucentric height, a working distance of $5 \mathrm{~mm}$ is required. The entire manipulator (total height: $52 \mathrm{~mm}$ ) fits underneath the final lens and is screwed onto the default SEM stage. An additional adapter block facilitates installation and removal of wiring. (c) Schematic diagram of the nanomanipulator setup.

find a suitable nano-object to be mounted. The sizes of samples that can be accommodated onto our manipulator range up to $30 \times 30 \mathrm{~mm}^{2}$ with a maximum height of $10 \mathrm{~mm}$.

A drawback of a system with a very large range often is its poor positioning accuracy. To take advantage of a large range and a high positioning accuracy, a coarse positioning system used for the approach was combined with a separate fine positioning system. For the coarse stage, a system consisting of three stackable positioners was used (Attocube, ANP 50 series, ANC 150 step controller), all nonmagnetic and UHV compatible. The fine stage, a flexure hinge design, is operated using piezostacks (PI, PICMA P-883.50, from -20 to $+120 \mathrm{~V}$ ) with a continuous range of motion. The piezostacks are integrated into the flexure hinges in a way that limits shear stress on the piezostacks, such that these stacks do not break if forces act laterally on them. Due to this design, coupling between the two mutually orthogonal directions of motion is avoided as $x$ and $y$ motions are integrated into the same body of material. Furthermore, this design enables easy installation of an actuator if replacement would be needed.

Separation of fine and coarse motion on two different stages allows accurate imaging of the tip and overcomes problems arising from unwanted motion during coarse positioning, such as hysteresis and vibrations of the stick-slip motor, which are discussed in detail in the supplementary material. ${ }^{6}$ When changing the step direction of a coarse stage actuator, it needs several step actuations before it is running properly in the indicated direction. In between, a combination of two unwanted effects is observed: motion in the opposite direction (which accumulates to a total of $\sim 300 \mathrm{~nm}$ ) and motion in the orthogonal directions (which accumulates to a total of $\sim 700 \mathrm{~nm}$ ). We attribute these effects to the reorientation of the rod-shaped piezo inside its housing after a step-direction change has been given, as the housing is clamped mechanically using springs onto the piezo over which it runs forward and backward. We find that it is not possible to use the coarse stage to perform accurate positioning processes. However, the magnitude of these effects is an order of magnitude smaller than the range of the fine stage and simple approach and retract operations can repeatedly be performed without any tip crashes, as the fine stage is designed to be robust and incorporating a large range.

\section{NANOMANIPULATOR OPERATION}

The coarse stage has a range of motion of $4 \mathrm{~mm}$ in $x$ and $y$ and $2.5 \mathrm{~mm}$ in $z$. The step sizes of the coarse stage are controlled by varying the driver signal amplitude and are specified to range from 25 to $500 \mathrm{~nm}$. The step size depends on the clamping force which is set by the manufacturer as well as on the mass that is being moved and the state of the sliding surfaces. ${ }^{7}$ Hence the step size as a function of driving signal amplitude will vary for each actuator. When operating the coarse stage at a $10 \mathrm{~V}$ actuation amplitude, which represents a compromise between minimal step size and reasonable reliability, this yields an average step size of approximately $90 \mathrm{~nm}$ in $x$ and $170 \mathrm{~nm}$ in $y$. Although the step sizes of the coarse stage actuators are not constant, as was discussed above, this does not interfere with any of the experiments we perform due to the fine stage design.

The fine stage allows continuous motion within a range of $15 \mu \mathrm{m}$ in $x, y$, and $z$. It is operated by custom-built piezodrivers which receive an input signal from a digital-toanalog converter (DAC) inside the PC. Motion in $x$ and $y$ are orthogonal within $1^{\circ}$, which is shown in the data in the supplementary material. ${ }^{6}$ The $z$ motion is not completely decoupled from the $y$ motion, probably due to the use of two piezoactuators that are not completely balanced. Over the entire range of motion of the fine stage $z$ piezo $(15 \mu \mathrm{m})$ the stage moves by $450 \mathrm{~nm}(3 \%)$ in the perpendicular directions, which can be compensated for by the piezocontrol software. When changing the direction of movement, the fine stage does not show overshoot in the wrong direction. An example of the fine stage operation during the process of mounting a multiwalled carbon nanotube (MWNT) is presented in a movie which can be viewed online, see Fig. 2.

Using LABVIEW, a user-interface was created that can be controlled using a three axes joystick system (Saitek, X52). The speed with which either the coarse or the fine stage moves, is determined by the joystick and can be adjusted to be more or less sensitive on the joystick motion. The joysticks are also used to switch between coarse and fine posi- 


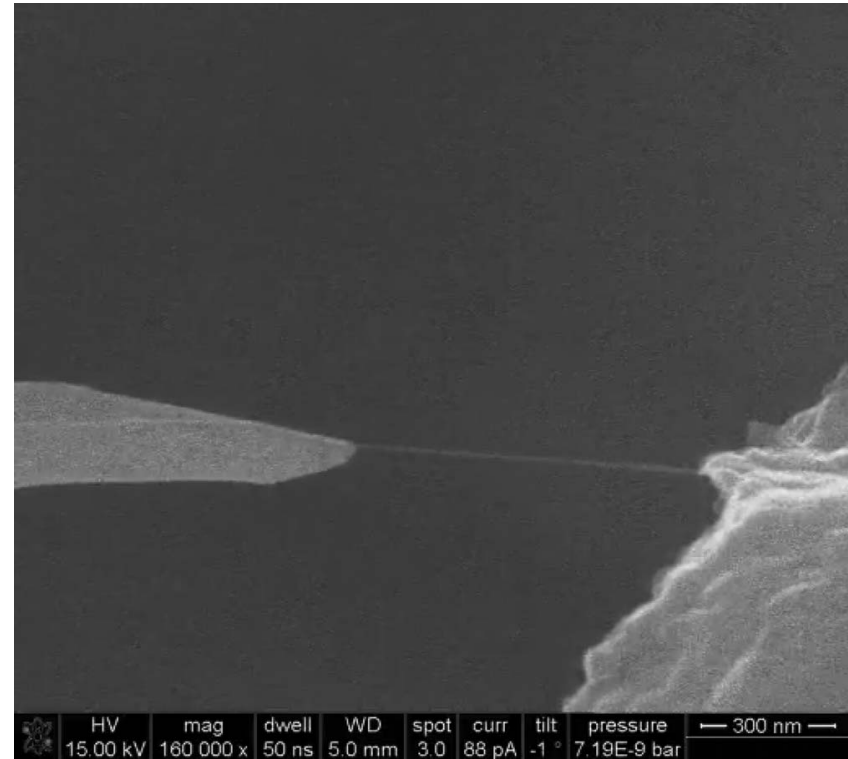

FIG. 2. The process of pulling a MWNT from its as-grown material by using a sharply etched tungsten tip mounted onto the fine stage. After approaching and attaching the MWNT (not shown) the tip is carefully retracted; movement is performed only by operating the fine stage. The substrate with the MWNT material is mounted onto the coarse (approach) stage. The still image shows a screen shot at $40 \mathrm{~s}$ (enhanced online). [URL: http://dx.doi.org/10.1063/1.3271033.1]

tioning and to apply single step actuations, voltage pulses, etc.

To perform $I-V$ measurements and in situ field emission tests of mounted carbon nanotubes, the sample and tip stages were electrically isolated from the base of the manipulator and wired to high-voltage connectors and feedthroughs. All wires can be disconnected from the SEM stage after which the manipulator can be removed within a few minutes for normal SEM imaging. In Fig. 1(c) a schematic diagram of the manipulator system is depicted. To prevent damaging the piezoactuators during venting or evacuating of the SEM, an interlock system was designed. Hence the manipulator can be operated only at ambient pressure and pressures below $10^{-4}$ mbar.

\section{APPLICATIONS}

As is shown by some examples, our design will work for a large variety of applications. To fabricate novel electron field emitters, both single-walled and MWNTs and semiconductor nanowires have been mounted. ${ }^{8,9}$ Closed MWNTs were mounted by pulling them from a sample with agglomerates of carbon nanotubes, see Fig. 2. We have managed to repeatedly mount single MWNTs with their as-grown cap by pulling the entire MWNT without breaking it from its asgrown material. ${ }^{10}$ In these experiments a long fine stage range is needed to be able to handle flexible nanotubes or nanowires. With the fine stage it is possible to manipulate micrometer-sized as-grown nanotubes and wires, without running out of range. Not only field emitters, but also highaspect ratio AFM-tips with carbon nanotubes have been created, which enable the studies of rough surfaces in liquid. ${ }^{11-13}$ In such mounting processes, nanotubes were cut using a voltage pulse, or by electron beam etching which was

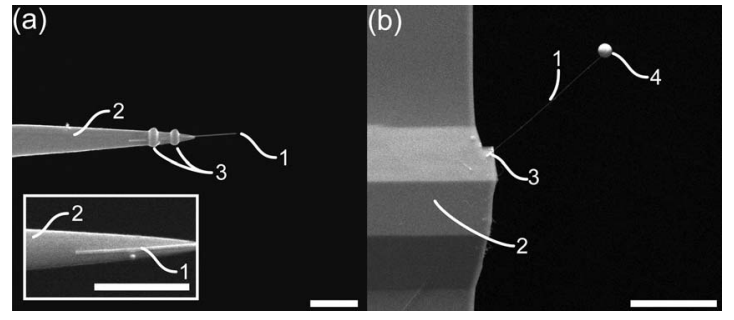

FIG. 3. Two examples of probes fabricated with the nanomanipulator. (a) InAs nanowire (1) after mounting on a sharply etched tungsten tip (2). Using EBID a layer of Pt (3) was deposited maskless at two positions to ensure a proper fixation of the nanowire onto the tip. The inset shows the tungsten tip with the nanowire before deposition. (Scale bars: $1 \mu \mathrm{m}$ ) (b) SiC nanowire (1) mounted on an AFM chip (2). After fixing the nanowire by EBID (3), a small magnetic $(\mathrm{NdFeB})$ particle (4) was added to the very end of the nanowire tip, also by EBID. In this way novel, very sensitive MRFM cantilevers can be constructed (scale bar: $30 \mu \mathrm{m}$ ).

facilitated by adding water vapor in the chamber. ${ }^{14}$ Using a GIS attached to our SEM, fixation of the nanotube or wire was improved by electron beam-induced deposition (EBID) of platinum at the position of overlap, see Fig. 3(a). Novel nanometer-sized electrochemistry electrodes consisting of an insulating AFM tip and mounted carbon nanotube were also created to study the electrochemistry properties of substances on a very small scale. ${ }^{15}$ A combination of techniques mentioned before, yields a very sensitive magnetic resonance force microscopy (MRFM) cantilever, see Fig. 3(b).

Besides mounting, the nanomanipulator is used as a positioning tool. It has been used to pick up nano-objects and put them down somewhere else, as has been demonstrated for diamond nanocrystals. ${ }^{16}$ The initial placement of a nanometer-sized object can be performed with an accuracy of about $20 \mathrm{~nm}$. After placement, the positioning accuracy can be enhanced by pushing the object with the tip. This final positioning is limited only by SEM imaging resolution of about $1 \mathrm{~nm}$ as the fine stage has a continuous range. The procedure to position these crystals is shown in Fig. 4. The tungsten tip was etched in such a way as to yield a somewhat

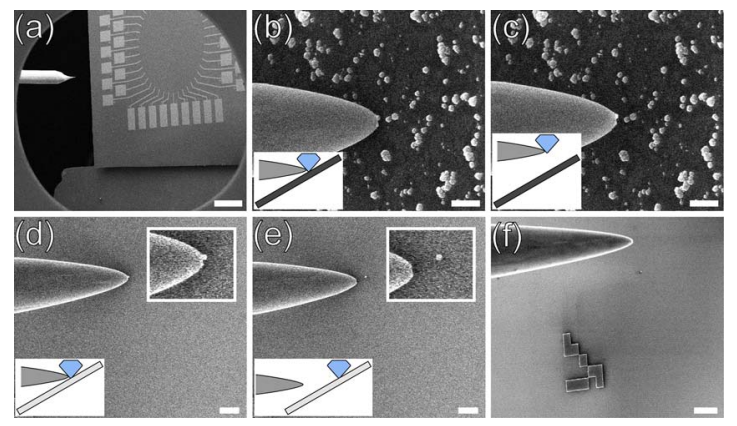

FIG. 4. (Color online) Positioning of a diamond nanocrystal. In this process a nanocrystal is picked up from a substrate onto which many were dispersed and positioned onto a different substrate containing markers located a few millimeters away. The insets in the lower left corners show a schematic representation of the position of the tip, substrate, and diamond. (a) Demagnified view of both substrates and the etched tungsten tip. Substrates are tilted to facilitate picking up and positioning of nanocrystals. (Scale bar: $500 \mu \mathrm{m})[(\mathrm{b})$ and (c)] Picking up the nanocrystal from the substrate (scale bars: $500 \mathrm{~nm})$. [(d) and (e)] Positioning near a marker on the other substrate (scale bars: $1 \mu \mathrm{m}$ ). (f) Demagnified view of (e), showing the positioned nanocrystal in the vicinity of a reference marker (scale bar: $2 \mu \mathrm{m}$ ). 
blunt, yet stiff and strong tip for the sole purpose to select and pick up the nanocrystals. Using a tip that was etched too sharply resulted in deformation of the tip as some nanocrystals were stuck to the sample very tightly. To determine whether or not the tip touches the surface, a bias voltage was set between tip and sample and the current was measured using a picoammeter (Keithley). In order to reposition nanometer-sized objects it is important to create a situation in which the adhesion of the object - that was picked up-to the tip_-with which it was picked up-is smaller than the adhesion to the surface onto which the object will be put down. This can be achieved by a combination of the following strategies. We try to keep the contact area between the object that is to be repositioned and the tip as small as possible. When the object is put down one can try to roll the object, in effect wiping it off the tip. It is also possible to put the object against another object and scrape it off the tip. Finally, we can use electron-induced deposition to fixate the manipulated object to the surface onto which it is to be deposited. Another strategy to facilitate manipulation is to apply a voltage difference across the sample and the tip. The detection of a current makes it easier to navigate the tip toward the surface. Additionally, the current through the manipulated object can be used to "weld" it to the tip or the surface onto which it is to be repositioned. Subsequent $I-V$ measurements can be used to characterize the quality of the electrical connections, which can be useful, e.g., for subsequent field emission experiments.

\section{CONCLUSION}

In conclusion, we have presented a stable and compact nanomanipulator consisting of a coarse stage with a range in $x, y$, and $z$ of several millimeters and a fine stage with a continuous range of $15 \mu \mathrm{m}$ in all three dimensions. Its use has been demonstrated already in a wide range of experiments. Vibrations are limited to approximately $10 \mathrm{~nm}$ except during coarse stage actuation. Further improvements on a new coarse stage design are in progress.

\section{ACKNOWLEDGMENTS}

We acknowledge support from STW and the Dutch Ministry of Economic Affairs and from L. Crama for experimental help.

${ }^{1}$ N. de Jonge, Y. Lamy, and M. Kaiser, Nano Lett. 3, 1621 (2003).

${ }^{2}$ D. Nakabayashi, P. C. Silva, and D. Ugarte, Appl. Surf. Sci. 254, 405 (2007).

${ }^{3}$ K. Andersen, K. Carlson, D. H. Petersen, K. Mølhave, V. Eichhorn, S. Fatikow, and P. Bøggild, Microelectron. Eng. 85, 1128 (2008).

${ }^{4}$ M. S. Fuhrer, J. Nygård, L. Shih, M. Forero, Y. G. Yoon, M. S. C. Mazzoni, H. J. Choi, J. Ihm, S. G. Louie, A. Zettl, and P. L. McEuen, Science 288, 494 (2000).

${ }^{5}$ T. Brintlinger, Y. F. Chen, T. Dürkop, E. Cobas, M. S. Fuhrer, J. D. Barry, and J. Melngailis, Appl. Phys. Lett. 81, 2454 (2002).

${ }^{6}$ See supplementary material at http://dx.doi.org/10.1063/1.3271033 for the pdf document containing the additional information about the coarse and fine stage.

${ }^{7}$ C. Meyer, O. Sqalli, H. Lorenz, and K. Karrai, Rev. Sci. Instrum. 76, 063706 (2005).

${ }^{8}$ N. de Jonge, Y. Lamy, K. Schoots, and T. H. Oosterkamp, Nature (London) 420, 393 (2002).

${ }^{9}$ E. C. Heeres, E. P. A. M. Bakkers, A. L. Roest, M. Kaiser, T. H. Oosterkamp, and N. de Jonge, Nano Lett. 7, 536 (2007).

${ }^{10}$ E. C. Heeres, N. de Jonge, and T. H. Oosterkamp (unpublished).

${ }^{11}$ M. H. van Es, Ph.D. thesis, Leiden University, 2008.

${ }^{12}$ A. J. Katan and T. H. Oosterkamp, J. Phys. Chem. C 112, 9769 (2008).

${ }^{13}$ H. Dai, J. H. Hafner, A. G. Rinzler, D. T. Colbert, and R. E. Smalley, Nature (London) 384, 147 (1996).

${ }^{14}$ T. D. Yuzvinsky, A. M. Fennimore, W. Mickelson, C. Esquivias, and A. Zettl, Appl. Phys. Lett. 86, 053109 (2005).

${ }^{15}$ A. V. Patil, A. F. Beker, F. G. M. Wiertz, H. A. Heering, G. Coslovich, R. Vlijm, and T. H. Oosterkamp (unpublished).

${ }^{16}$ T. van der Sar, E. C. Heeres, G. M. Dmochowski, G. de Lange, L. Robledo, T. H. Oosterkamp, and R. Hanson, Appl. Phys. Lett. 94, 173104 (2009). 